\title{
Thermoelastic Problems of Multilayered Spherical Pressure Vessels Subjected to Axisymmetric Loading
}

\author{
D. GÖNCZI \\ University of Miskolc, Institute of Applied Mechanics, mechgoda@uni-miskolc.hu
}

\begin{abstract}
This paper deals with the linear thermoelastic analysis of functionally graded multilayered spherical bodies subjected to constant mechanical and thermal loading. The temperature field is arbitrary function of the radial coordinate, the material properties and the radial body force vary according to power law functions along the radius of the sphere. An analytical method is presented to calculate the displacements and stresses within the multilayered spherical body. The method is expanded to tackle the problem of spherical bodies made from radially graded materials with temperature dependent material properties. The results are compared to finite element simulations and other methods.
\end{abstract}

Keywords: FGM, thermoelastic, sphere, multilayered

\section{Introduction}

In recent years, laminated or multilayered composite and functionally graded materials (or FGMs for short) have been widely used in numerous engineering applications due to their excellent material properties. The concept of FGM was first considered in Japan in the 1980's during a hypersonic space plane project, where the body of the spaceplane is exposed to an extremely high temperature environment (about $2000 \mathrm{~K}$ ), with a large temperature gradient (approximately $1000 \mathrm{~K}$ ), between the inside and outside of the spaceplane which required an advanced material. In a functionally graded material the composition and structure gradually change, resulting in a corresponding change in the material properties. Due to the smooth transition between the constituent components, these materials have improved thermal resistance and mechanical properties. In recent decades this concept has become more popular, a lot of textbooks [1-3] and papers deal with the determination of stresses and displacements within simple structural components (e.g. spherical bodies, beams or disks) caused by steady-state temperature field and mechanical loading.

Papers, such as [4-10] investigate heterogeneous and functionally graded beams from different aspect. Works by Pen [11], Gönczi [12-13], Sondhi [14] and Allam [15] (etc.) dealt with the thermomechanical problems of disks subjected to thermal and mechanical loads.

Paper [16] studied the elastic and perfectly plastic radially graded spheres where the material properties were power functions of the radial coordinate.

Kar and Kanoria [17] dealt with the determination of thermoelastic interaction due to a step thermal loading on the boundary surfaces of a radially graded orthotropic spherical body, while paper [18] 
presented an analytical approach to investigate the non-linear response of radially graded shallow spherical shells subjected to axisymmetric external pressure and temperature field incorporating the effects of imperfections. Paper [19] gives solutions for radially graded cylinders and spheres where the material properties were described by specific exponential functions. Nayak et. al in [20] give analytical solutions to determine the stress and displacement fields within thick-walled functionally graded spherical pressure vessels. Paper [21] presented a method to determine the stresses within radially graded spherical bodies where the material properties were arbitrary functions of the radial coordinate and temperature field based on a multi-layered approach. Akinlabi et. al. [22] investigated a thermoelastoplastic method to analyse radially graded spheres after thermal treatment.

In this paper, our objective is to develop a method to determine the exact stresses and displacements in multilayered spherical bodies subjected to thermal loading, constant pressure and incorporating radial body forces. The material properties and the radial body force are considered to be power functions of the radial coordinate (in $r \varphi \vartheta$ spherical coordinate system) within the layers, the number of layers is denoted by $N$. The constant pressure is denoted by $p_{\text {outer }}$ which acts on the outer curved boundary surface $\left(r=R_{N+1}\right)$ while the uniformly distributed mechanical loading exerted on the inner surface $\left(r=R_{1}\right)$ is denoted by $p_{\text {inner }}$. The temperature field $T(r)$ is arbitrary function of the radial coordinate. The $i$-th layer is illustrated in Fig. 1. The method can be modified to tackle the problems of radially graded spherical pressure vessels where the material properties, temperature field and the radial body force are arbitrary functions of the radial coordinate furthermore the material properties depend on the temperature field.

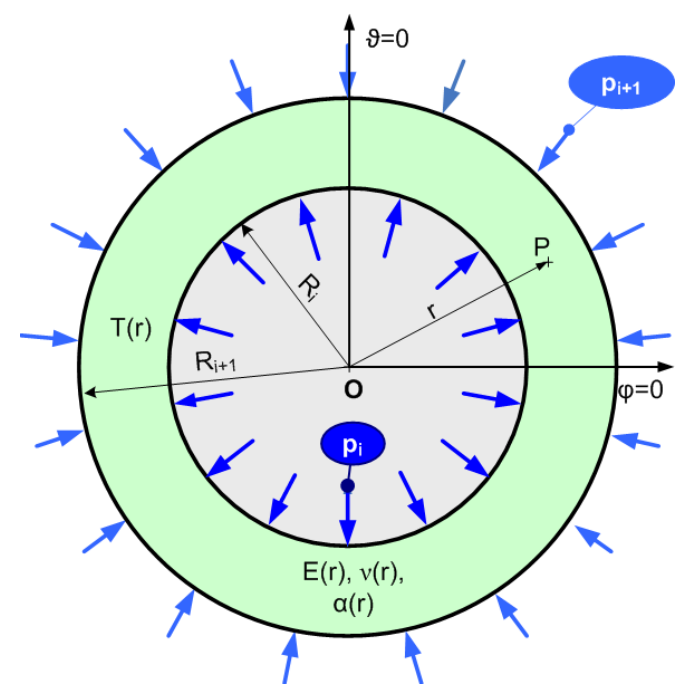

Figure 1. The sketch of the i-th layer.

\section{Formulation of the problem}

For this one-dimensional problem the non-zero coordinate of the displacement field (in the radial direction) can be used to construct the kinematic equation as

$$
\varepsilon_{r, i}=\frac{\mathrm{d} u_{r, i}(r)}{\mathrm{d} r}
$$




$$
\varepsilon_{\varphi, i}=\frac{u_{r, i}}{r}, \quad r=R_{i} \ldots R_{i+1},
$$

in the $i$-th layer. For isotropic materials the constitutive equations (Hooke's law for isotropic linearly elastic materials) are

$$
\begin{gathered}
\sigma_{r, i}=\frac{E_{i}}{\left(1+v_{i}\right)\left(1-2 v_{i}\right)}\left[\left(1-v_{i}\right) \varepsilon_{r, i}+2 v_{i} \varepsilon_{\varphi, i}-\left(1+v_{i}\right) \alpha_{i} \vartheta\right], \\
\sigma_{\varphi, i}=\sigma_{\theta, i}=\frac{E_{i}}{\left(1+v_{i}\right)\left(1-2 v_{i}\right)}\left[v_{i} \varepsilon_{r, i}+\varepsilon_{\varphi, i}-\left(1+v_{i}\right) \alpha_{i} \vartheta\right] .
\end{gathered}
$$

In this case the equilibrium equation is

$$
\frac{\mathrm{d} \sigma_{r, i}}{\mathrm{~d} r}+\frac{2}{r}\left(\sigma_{r, i}-\sigma_{\varphi, i}\right)+q_{r, i}=0,
$$

Let the material properties (except Poisson's ratio) and the body force be

$$
\begin{gathered}
E_{i}(r)=E_{0, i}\left(\frac{r}{R_{i}}\right)^{m, i}, \alpha_{i}(r)=\alpha_{0, i}\left(\frac{r}{R_{i}}\right)^{m, i}, \\
q_{r, i}(r)=b_{i} r^{n_{i}},
\end{gathered}
$$

where $E_{0, i}, \alpha_{0, i}, m, n, b_{i}$ are constants. The combination of the kinematic, constitutive and equilibrium equations (1-6) results in a differential equation for the displacement field. After some manipulation, the homogeneous part of the solution is

$$
u_{h, i}(r)=A_{i} r^{0.5 \frac{\left(m_{i}+1\right)\left(1-v_{i}\right)+C_{2, i}}{v_{i}-1}}+B_{i} r^{-0.5 \frac{\left(m_{i}+1\right)\left(-1+v_{i}\right)+C_{2, i}}{v_{i}-1}},
$$

where $A_{i}$ and $B_{i}$ are unknown constants of integration for the $i$-th layer, furthermore

$$
\begin{gathered}
C_{1, i}=\sqrt{\left(D_{1, i} v_{i}-D_{3, i}\right)\left(v_{i}-1\right)}, \quad C_{2, i}=\sqrt{D_{1, i} v_{i}^{2}+D_{2, i} v_{i}+D_{3, i}} \\
D_{1, i}=m_{i}^{2}+10 m_{i}+9, \quad D_{2, i}=-2 m_{i}^{2}-12 m_{i}-18, \quad D_{3, i}=m_{i}^{2}+2 m_{i}+9 .
\end{gathered}
$$

The inhomogeneous part can be expressed as

$$
u_{i n, i}(r)=\frac{-v_{i}-1}{C_{1, i} E_{0, i}}\left(r^{\frac{C_{1}+\left(m_{i}+1\right)\left(1-v_{i}\right)}{2\left(v_{i}-1\right)}} I_{1, i}-r^{\frac{-C_{1}+\left(m_{i}+1\right)\left(1-v_{i}\right)}{2\left(v_{i}-1\right)}} I_{2, i}\right),
$$

where

$$
\begin{gathered}
I_{1, i}=\int_{R_{i}}^{r}\left[2\left(v_{i}-0.5\right) b_{i}\left(\frac{\rho}{R_{i}}\right)^{-m_{i}} \rho \frac{-C_{1, i}+\left(3+m_{i}+2 n_{i}\right)\left(v_{i}-1\right)}{2\left(v_{i}-1\right)}+2\left(\frac{\rho}{R_{i}}\right)^{m_{i}} \alpha_{o, i} E_{0, i} \tau_{1, i}(\rho)\right] \mathrm{d} \rho \\
\tau_{1, i}(r)=r^{\frac{-C_{1, i}+\left(m_{i}+1\right)\left(v_{i}-1\right)}{2\left(v_{i}-1\right)}} m_{i} T(r)+0.5 \frac{\mathrm{d} T(r)}{\mathrm{d} r} r \frac{-C_{1, i}+\left(m_{i}+3\right)\left(v_{i}-1\right)}{2\left(v_{i}-1\right)}
\end{gathered}
$$

and

$$
I_{2, i}=\int_{R_{i}}^{r}\left[2\left(v_{i}-0.5\right) b_{i}\left(\frac{\rho}{R_{i}}\right)^{-m_{i}} \rho \frac{C_{1, i}+\left(3+m_{i}+2 n_{i}\right)\left(v_{i}-1\right)}{2\left(v_{i}-1\right)}+2\left(\frac{\rho}{R_{i}}\right)^{m_{i}} \alpha_{o, i} E_{0, i} \tau_{2, i}(\rho)\right] \mathrm{d} \rho
$$




$$
\tau_{2, i}(r)=r \frac{C_{1, i}+\left(m_{i}+1\right)\left(v_{i}-1\right)}{2\left(v_{i}-1\right)} m_{i} T(r)+0.5 \frac{\mathrm{d} T(r)}{\mathrm{d} r} r \frac{C_{1, i}+\left(m_{i}+3\right)\left(v_{i}-1\right)}{2\left(v_{i}-1\right)}
$$

The general solution for the $i$-th layer is

$$
u_{i}(r)=u_{h, i}(r)+u_{i n h, i}(r) .
$$

To calculate the unknown constants of Eq. (12), the boundary and fitting conditions can be used. There are 2 unknown constants for every layer $\left(A_{i}, B_{i}, i=1 \ldots N\right)$, which means that we need $2 N$ equations to determine them. The normal stresses can be calculated according to Eqs. $(3,4)$, furthermore the radial displacement field and the radial normal stresses are continuous. To ensure the continuity, we can express the following fitting conditions between the layers:

$$
\begin{gathered}
u_{i}\left(R_{i+1}\right)=u_{i+1}\left(R_{i+1}\right), \quad \\
\sigma_{r, i}\left(R_{i+1}\right)=\sigma_{r, i+1}\left(R_{i+1}\right), \quad i=1 \ldots N-1 .
\end{gathered}
$$

Additionally, the boundary conditions at the inner and outer spherical boundary surfaces can be (for example)

$$
\sigma_{r, 1}\left(R_{1}\right)=-p_{\text {inner }}, \quad \sigma_{r, N}\left(R_{N+1}\right)=-p_{\text {outer }},
$$

or with kinematic boundary conditions

$$
u_{1}\left(R_{1}\right)=-u_{\text {inner }}, \quad u_{N}\left(R_{N+1}\right)=-u_{\text {outer }},
$$

or the combination of these boundary equations. With these equations we have $2 \mathrm{~N}$ equations to calculate $2 N$ unknown constants of integrations.

\section{Radially graded spherical bodies}

Consider a radially graded and/or multilayered spherical body, in which the material properties are arbitrary functions of the radial coordinate and temperature field, furthermore the body force and the temperature field are given arbitrary functions of the radial coordinate.

$$
E(r, T), \alpha(r, T), v(r, T), b(r), T(r) .
$$

To solve this problem, a multilayered approach is used based on the previously derived equations. The sphere is divided into $N$ sublayers, in which the conditions described in section 1 are valid. We can use a method to approximate the original material distribution with the sublayers. The functions used in Eqs. (6) have 2 parameters, which means that we can us for example an integral average sense or the material property values at the boundaries of the layers $\left(R_{i}, R_{i+1}\right)$. The temperature field is given, the temperature dependency can be eliminated by substituting a value into the function of the material property. In the simplest case the temperature value at the middle of the layer can be used. For the Young's modulus we have

$$
E\left(R_{i}, T_{m}\right)=E_{0, i}, \quad E\left(R_{i}, T_{m}\right)=E_{0, i}\left(\frac{R_{i+1}}{R_{i}}\right)^{m, i}, \quad T_{m}=T\left(\frac{R_{i}+R_{i+1}}{2}\right),
$$

from which we can determine the $E_{0, i} ; m, i$ constants for every layer. For the linear thermal expansion we can use the same approach, while for the Poisson's ratio 


$$
v(r, T) \rightarrow v_{i}\left(\frac{R_{i}+R_{i+1}}{2}, T_{m}\right)
$$

we have a constant value for every subdomain.

A similar approach can be used to determine the approximation of the body force:

$$
q_{r}\left(R_{i}\right)=b_{i} r^{n_{i}}, \quad q_{r}\left(R_{i+1}\right)=b_{i} r^{n_{i}} \rightarrow b_{i}, n_{i}, i=1 \ldots N . \text { (20) }
$$

With these parameters, the problem can be solved. Obviously, with the increase of the number of layers, the error decreases.

\section{Numerical examples}

In our first numerical example, we have one spherical layer with the following data:

$$
\begin{gathered}
N=1, R_{1}=0.5 \mathrm{~m}, R_{2}=0.6 \mathrm{~m}, v=0.3, E_{0}=2 \cdot 10^{11} \mathrm{~Pa}, \alpha_{0}=1.2 \cdot 10^{-6} \frac{1}{\mathrm{~K}}, m=3, \\
p_{\text {inner }}=50 \mathrm{MPa}, p_{\text {outer }}=0 \mathrm{MPa}, T(r)=-400+3500 r^{3}(\mathrm{~K}) .
\end{gathered}
$$

The finite element model was created in Abaqus [23] as an axisymmetric quarter sphere built from 20 homogeneous, isotropic layers with material distribution Eq. (19) [21]. The layers are perfectly bonded, coupled temperature-displacement element type was used. In the first case, the body force is negligible, which means that $b=0$. In this case, the equations presented in [21] can be used to verify the results too. Figure 2 shows the displacement, Fig. 3 plots the stress distributions along the radial coordinate.

The results were in good agreement with the results of the FE simulation (Fig. 2) and of the alternative method presented in [21]. The comparison of the dominant hoop stresses can be in Fig. 3, where the curve fitting method of [21] was used to smoothen the distribution of the stresses.

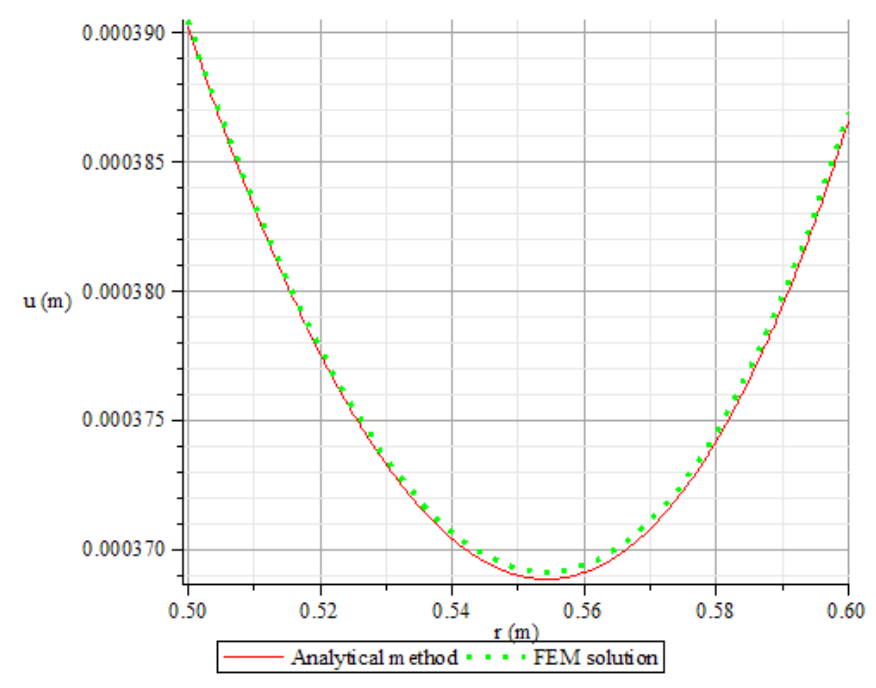

Figure 2. The radial displacement of the first case. 

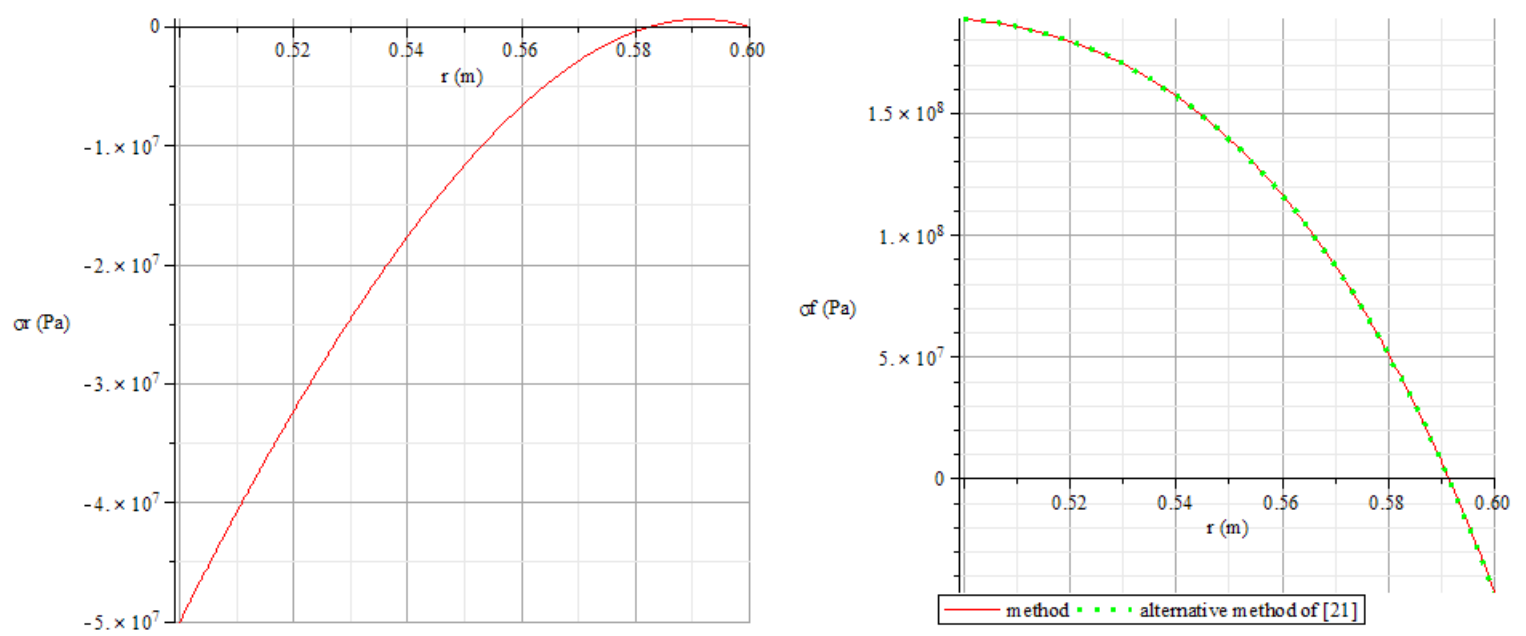

Figure 3. The normal stresses of the first case.

In our second case we added a non-zero body force with $b_{0}=2 \cdot 10^{8}, n=2$. Figures 4 and 5 shows the results for the displacement and stress fields, which were in good agreement with the FE simulations.

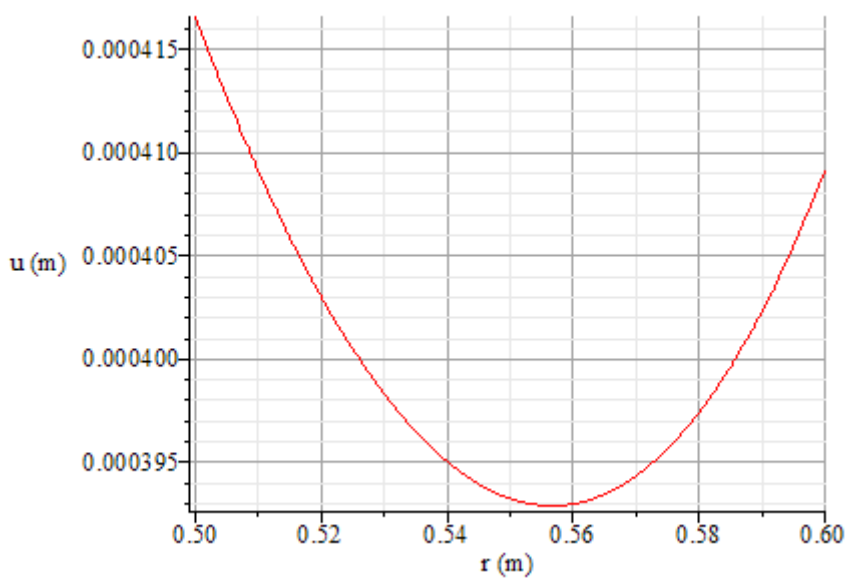

Figure 4. The radial displacement of the second case. 

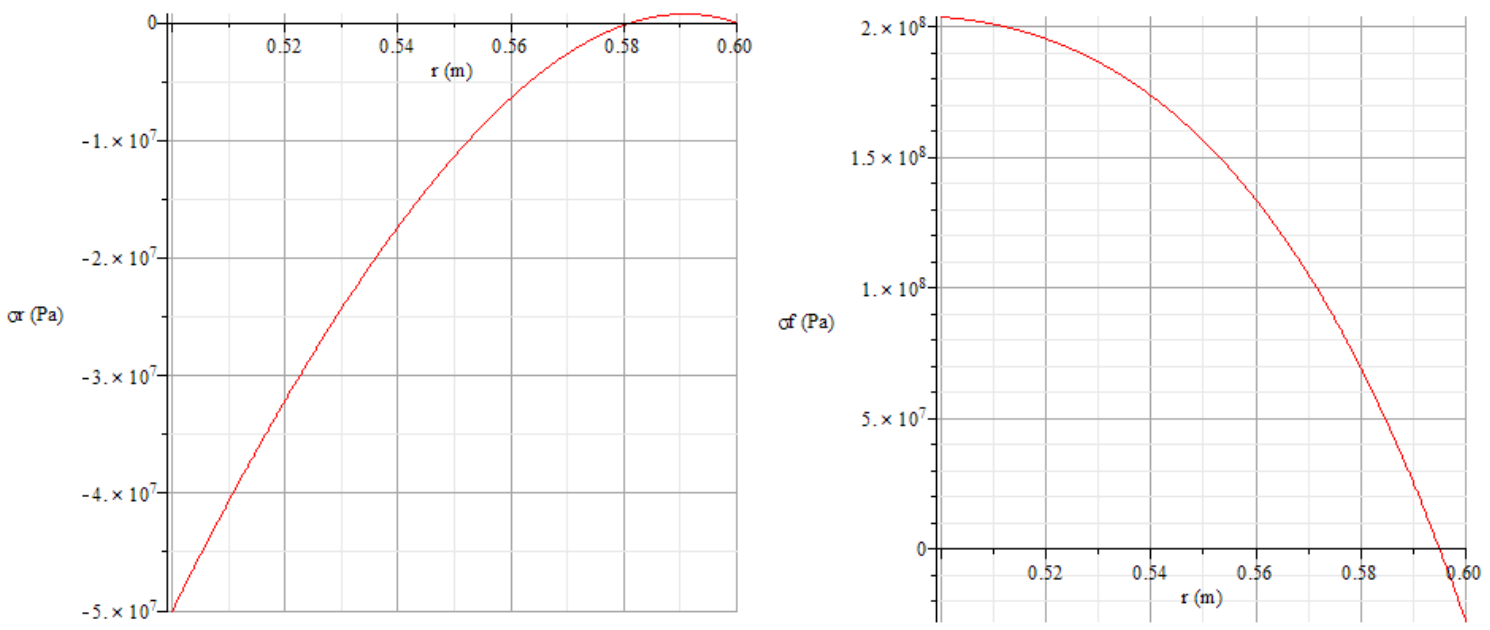

Figure 5. The normal stresses of the second case.

In our last example we considered a stainless steel - silicon nitride functionally graded material.

$$
R_{1}=0.5 \mathrm{~m}, R_{2}=0.59 \mathrm{~m}, m=3, p_{\text {inner }}=100 \mathrm{MPa}, p_{\text {outer }}=0 \mathrm{MPa}, T_{\text {inner }}=303 \mathrm{~K}, T_{\text {outer }}=733 \mathrm{~K} \text {, }
$$

the distributions of the material properties are [24]:

$$
\begin{array}{r}
M(r, T)=\left(M_{m}-M_{c}\right)\left(\frac{r-R_{1}}{R_{2}-R_{1}}\right)^{m}+M_{c}, \\
M_{j}=P_{j 0}\left(1+P_{j 1} T+P_{j 2} T^{2}+P_{j 3} T^{3}\right), \quad j=m, c,
\end{array}
$$

where Table 1 shows the material parameters.

\begin{tabular}{|c|c|c|c|c|c|c|c|c|}
\hline \multirow{2}{*}{ material } & \multicolumn{4}{|c|}{ metal (stainless steel) } & \multicolumn{3}{c|}{ ceramic (silicon nitride) } \\
\cline { 2 - 9 } & $P_{\mathrm{m} 0}$ & $P_{\mathrm{m} 1}\left(10^{-3}\right)$ & $P_{\mathrm{m} 2}\left(10^{-7}\right)$ & $P_{\mathrm{m} 3}\left(10^{-10}\right)$ & $P_{c 0}$ & $P_{\mathrm{c} 1}\left(10^{-3}\right)$ & $P_{\mathrm{c} 2}\left(10^{-7}\right)$ & $P_{\mathrm{c} 3}\left(10^{-11}\right)$ \\
\hline$\lambda(\mathrm{W} / \mathrm{mK})$ & 15.39 & -1.264 & 20.92 & -7.223 & 12.723 & -1.032 & 5.466 & -7.876 \\
\hline$\alpha(1 / \mathrm{K})$ & $12.33 \cdot 10^{6}$ & 0.8086 & 0 & 0 & $3.873 \cdot 10^{-6}$ & 0.9095 & 0 & 0 \\
\hline $\mathrm{E}(\mathrm{Pa})$ & $2.01 \cdot 10^{11}$ & 0.3079 & -6.534 & 0 & $3.484 \cdot 10^{11}$ & -0.307 & 2.16 & -8.946 \\
\hline $\mathrm{V}(-)$ & 0.3262 & -0.1 & 3.797 & 0 & 0.24 & 0 & 0 & 0 \\
\hline
\end{tabular}

Table 1. The material properties for the two-component FGM.

Figure 6 shows the results of the stress fields (with curve fitting with $N=50$ layers) which are in good agreement in the results of [21]. 


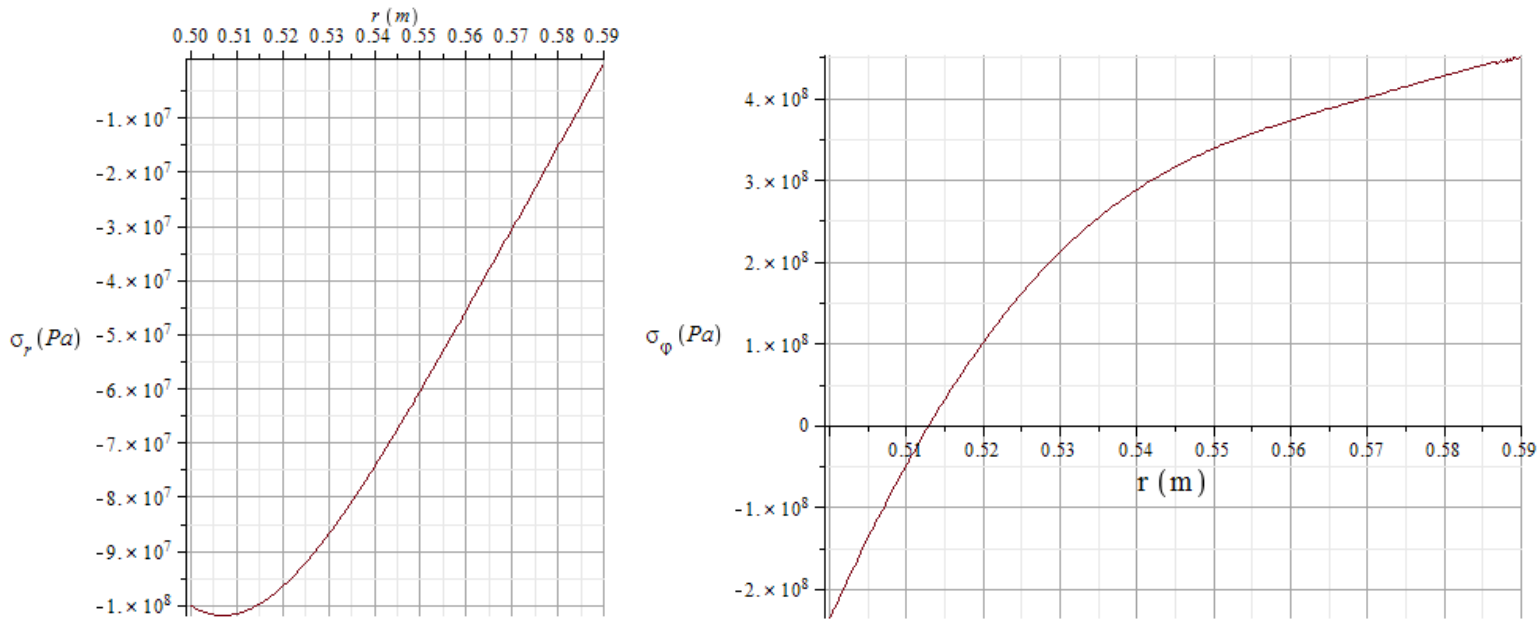

Figure 6. The normal stresses of the third case.

\section{Conclusions}

An analytical method was developed to determine the displacement and stress fields within a multilayered spherical pressure vessel subjected to combined mechanical and thermal loads based on the equations of thermoelastostatics. The material properties and the radial body force within the layers are specific power functions of the radial coordinate (except the Poisson's ratio), the steady-state temperature field is an arbitrary function of the radial coordinate. The method is modified to deal with the thermoelastic problems of radially graded, multilayered spherical bodies with temperature- and arbitrary radial coordinate dependent material properties. The results were verified by FE simulations and other methods of the literature.

\section{References}

[1] Boley, B. A. and Weiner, J. H. (1960) 'Theory of Thermal Stresses' John Wiley \& Sons Inc., New York,

[2] Lekhnitskii, S. G. (1981) 'Theory of Elasticity of an Anisotropic Body' Mir Publishers, Moscow

[3] Shen H-S. (2009) 'Functionally Graded Materials: Nonlinear Analysis of Plates and Shells' CRC Press, London

[4] Kiss, L.P., Szeidl, Gy. (2014) 'In-plane Buckling of Rotationally Restrained Heterogeneous Shallow Arches Subjected to a Concentrated Force at the Crown Point' Journal of Computational and Applied Mechanics, 9(2), pp. 171-199. doi: 10.1080/15397734.2015.1022659

[5] Gönczi, D., Ecsedi, I. (2015) 'Thermoelastic stresses in nonhomogeneous prismatic bars' Annals of Faculty of Engineering Hunedoara, 13(2), pp. 49-52.

[6] Kiss, L.P., Szeidl, Gy. (2015) 'Vibrations of pinned-pinned heterogeneous circular beams subjected to a radial force at the crown point' Mechanics Based Design of Structures and Machines, 43(4), pp. 424-449. doi: 10.1080/15397734.2015.1022659 
[7] Ecsedi, I., Baksa, A. (2017) 'A half circular beam bending by radial loads' Journal of Computational and Applied Mechanics, 12(1), pp. 3-18.

[8] Babaei, H., Kiani, Y., Eslami, M.R. (2019) 'Thermomechanical non-linear in-plane analysis of fixended FGM shallow arches on nonlinear elastic foundation using two-step perturbation technique' Int. J. Mech. Mater. Des., 15(2), pp. 225-244. doi: 10.1007/s10999-018-9420-y

[9] Gönczi, D. (2019) 'Analysis of curved bimetallic beam' Journal of Computational and Applied Mechanics, 41(1-2), pp. 41-51, 2019. doi: 10.32973/jcam.2017.008

[10] Hua, Y.-J., Zhoua, H., Zhub, W., Zhu J. (2020) 'A thermally-coupled elastic large-deformation model of a multilayered functionally graded material curved beam' Composite structures, 244: 112241, doi: 10.1016/j.compstruct.2020.112241

[11] Pen X., Li X. (2009) 'Thermoelastic analysis of functionally graded annulus with arbitrary gradient' Applied Mathematics and Mechanics (English Edition), 30 (10), pp. 1211-1220. doi: 10.1007/s10483-009-1001-7

[12] Gönczi, D., Ecsedi, I. (2015) 'Thermoelastic analysis of functionally graded hollow circular disc' Archive of Mechanical Engineering, 62 (1), pp. 5-18. doi: 10.1515/meceng-2015-0001

[13] Gönczi, D. (2018) 'Analysis of Rotating Functionally Graded Disks with Arbitrary Material Properties' Acta Technica Corviniensis - Bulletin Of Engineering, 11, pp. 1-6. Paper: 1.

[14] Sondhi L., Thawait AK., Sanyal S., Bhowmick S. (2019) 'Stress and Deformation Analysis of Variable Thickness Clamped Rotating Disk of Functionally Graded Orthotropic Material' Materials Today: Proceedings, 18(7), pp. 4431-4440. doi:10.2478/mme-2019-0027

[15] Allam MNM., Tantawy R., Zenkour AM. (2019) 'Thermoelastic stresses in functionally graded rotating annular disks with variable thickness' Journal of Theoretical and Applied Mechanics, 56(4), pp. 1029-1041. doi: 10.15632/jtam-pl.56.4.1029

[16] Sadeghian, M., Toussi, H.E. (2011) 'Axisymmetric yielding of functionally graded spherical vessel under thermo-mechanical loading' Computational Material Science, 50(3), pp. 975-981. doi: 10.1016/j.commatsci.2010.10.036

[17] Kar A., Kanoria M. (2009) 'Generalized thermoelastic functionally graded orthotropic hollow sphere under thermal shock with three-phase-lag effect' European Journal of Mechanics, A/Solids, 28(4), pp. 757-767. doi: 10.1016/j.euromechsol.2009.01.003

[18] Bich, D.H., Tung, H.V. (2011) 'Non-linear axisymmetric response of functionally graded shallow spherical shells under uniform external pressure including temperature effects' International Journal of Non-Linear Mechanics, 46(9), pp. 1195-1204. doi: 10.1016/j.ijnonlinmec.2011.05.015

[19] Chen, Y.Z., Lin, X.Y. (2008) 'Elastic analysis for thick cylinders and spherical pressure vessels made of functionally graded materials' Computational materials science, 44(2), pp. 581-587. doi: 10.1016/j.commatsci.2008.04.018

[20] Nayak, P., Mondal, S. C., Nandi A. (2011) 'Stress, strain and displacement of a functionally graded thick spherical vessel' International Journal of Engineering Science and Technology, 3(4), 26602671. 
[21] Gönczi, D. (2017) 'Thermoelastic analysis of thick-walled functionally graded spherical pressure vessels with temperature-dependent material properties' Journal of Computational and Applied Mechanics, 12(2), pp. 109-125. doi: 10.32973/jcam.2017.008

[22] Akinlabi, E. T., Dats, E. P., Murashkin, E. V. (2020) 'Thermoelasticplastic deformation of a functionally graded spherical layer' Journal of Physics: Conference series, 1474, 012002, doi: 10.1088/1742-6596/1474/1/012002

[23] Abaqus Standard User's Manual Version 6.13, 2017.

[24] Gönczi, D. (2016) 'Thermoelastic Problems of Functionally Graded and Composite Structural Components'. (Dissertation) 